\title{
Commentary: Pleiotropic Neuropathological and Biochemical Alterations associated with Myo5a Mutation in a Rat Model
}

\author{
George Stoica ${ }^{1 *}$, Heidi Martini-Stoica ${ }^{2}$ \\ 'Texas A\&M University, College Station, TX,USA \\ ${ }^{2}$ Baylor College of Medicine, Houston, TX, USA
}

\section{Article Info}

\section{Article Notes}

Received: November 9, 2018

Accepted: December 24, 2018

\section{*Correspondence:}

Dr. George Stoica, DVM, MSc., Ph.D., Professor Emeritus, Texas A\&M University, College Station, Texas, USA; E-mail: gstoica@cvm.tamu.edu.

(c) 2018 Stoica G. This article is distributed under the terms of the Creative Commons Attribution 4.0 International License
Molecular motors have a broad range of functions in the nervous system, including brain wiring, neuronal survival, neuronal plasticity, higher brain function, and control of central nervous system and peripheral nervous system development ${ }^{1}$. In Landrock et al., a rat model with neurodegeneration, movement disorder, and early death is associated with loss of function of the molecular motor protein Myosin5a (Myo5a $)^{2}$. Myo5a, highly expressed in the brain and skin and the predominant neuronal type, is an actin-based motor protein, which is part of a large group of unconventional myosins. Myosin5a plays a major role in cytoskeletal dynamics in the nervous system, potentially mediating movements of organelles and proteins in neurons ${ }^{3,4}$. However, little is known about the precise role of $M y o 5 a$ in neuronal function and survival. This study is the first to associate accumulation of tau$\mathrm{P} / \alpha$-syn and altered striatal dopamine metabolism with Myo5a loss of function ${ }^{2}$. Furthermore, prior studies of this same model demonstrate additional phenotypes including neuroendocrine abnormalities related to hypothyroidism and altered microRNA expression ${ }^{5,6}$. Indeed, Myo5a is responsible for pleiotropic systemic developmental effects, which underscores the importance of this molecular motor in the development of the central nervous system, and perhaps also in the pathogenesis of certain neurodegenerative disorders.

Our first report of this rat model, in which we were unaware of the Myo5a mutation, was published in $2007^{5}$ with two more publications to follow, where we were still unaware of Myo5a mutation ${ }^{6,7}$. However, from these reports we obtained significant pathological data, which helped us to better understand the complexity of this neurologic/ movement disorder, eventually identified as being associated with a mutation in $\mathrm{Myo5}^{2}$. These publications are interconnected and represent a continuation of the same neurologic/degenerative disorder cause by a Myo5a mutation in a rat model. In this article we comment on two pathological aspects that led us to perform whole-genome next generation sequencing (WGS) to determine the nature of the mutation that was associated with the neurologic phenotype we observed in our rat model. Those are as follows:

- Hypothyroidism neuroendocrine alterations

- Potential role of MicroRNA in Myo5a rat model of neurodegeneration

A brief historical perspective might be helpful for understanding the disease pathogenesis in this Myo5a mutant rat model. A spontaneous inherited autosomal recessive rat model for neurodegeneration was developed in our laboratory in a colony of Sprague-Dawley rats 
maintained at the Texas A\&M University Lab Animal Facility, USA ${ }^{5}$. Due to the fact that SD rats are an outbred strain, we transferred the movement disorder phenotype into a syngeneic black hooded rat, Berlin-Druckrey IV (BD-IV) by inbreeding. The affected BD-IV offspring can be identified during the first few days post-natal (dpn) by the gray color of their coat, which co-segregates with the dilute-opisthotonus phenotype ${ }^{8,9}$. The clinical phenotype is characterized by progressive development of tremor, spasticity and rigidity, bradykinesia, and postural instability, which are obvious by $15 \mathrm{dpn}$. Mutations in Myo5a have been shown to cause pigmentation and neurological defects in humans and animals. Mutation of human Myo5a causes Griscelli syndrome type 1 a rare autosomal recessive disorder ${ }^{10,11,12}$.

Mutations in horse Myo5a cause Lavender foal syndrome $^{13}$. Lavender foal syndrome (OMIA 001501-9796) is a recessive lethal disorder of Arabian foals characterized by a dilute coat color and a range of neurological signs, including recumbency, opisthotonus, padding movements, and extensor rigidity. The genetic basis is a single base pair deletion in the Myo5a gene that leads to a frame shift and premature stop codon ${ }^{13}$. Both the sire and the dam must be carriers of the specific gene that contributes to the disorder.

Myo5a is also mutated in dilute mice ${ }^{14}$. Investigations of dilute mice have suggested that myosin Va is important for melanosome transport in melanocytes and smooth endoplasmic reticulum trafficking in dendritic spines of Purkinje cells ${ }^{15}$. Most 'dilute' alleles also produce a neurologic defect characterized by convulsions and opisthotonus, apparent at 8 to 10 days of age and continuing until the death of the animal at 2 to 3 weeks of age. Mutation in Myo5a is also found in dilute opisthotonus rats ${ }^{9,16}$. Diluteopisthotonus is a spontaneous Myo5a gene mutation in the rat, and phenotypes of the homozygote (dop/dop) are similar to those of the myosin Va-deficient mouse. Ultrastructural study in dilute-opisthotonus rats revealed the absence of smooth endoplasmic reticulum and inositol 1,4,5-triphosphate receptors in the cerebellar Purkinje cell dendritic spines ${ }^{16,17}$. The comparison of the genetic maps of rat chromosome 8 and mouse chromosome 9 shares synteny indicating that the dop locus is homologous to the mouse dilute locus ${ }^{18}$.

The pigmentation defects of dilute myosin Va mutants in human and animal disease and their underlying mechanisms are well understood ${ }^{8}$. Our investigations on the neuroendocrine pathology associates with Myo5a mutation in a rat model underscores the complexity of the mechanisms implicated in neurological deficit and open the gate for future investigation in human and animal disease afflicted by this mutation.

In an initial report, we described the endocrine and neuropathological findings in Sprague-Dawley affected rats. The cerebellum was mostly affected by dystrophic dendrites, atrophy, and Purkinje cell loss. The microscopic appearance of CNS lesions suggested that the neuronal change is a "dying back" type of neuronal alteration, progressing from the dendrites to the perikaryon and nucleus 5 .

Importantly, in the second publication using the BD-IV syngeneic rat strain in which the mutated phenotype was transferred by inbreeding from SD outbred rat strain we document the presence of perikaryal and neuritic Lewy bodies in the basal ganglia as well as a progressive increase in $\alpha$-synuclein content in the mesencephalon followed by loss of dopaminergic terminals in the striatum. The severity of the pathology correlates with the upregulation of $\alpha$-synuclein and a parallel decrease in dopamine levels in the striatum of the affected rats. The disease process is accompanied by gliosis and release of inflammatory cytokines ${ }^{7}$.

In the third report we found that some microRNAs (miRs) were dysregulated in the affected mutant BD-IV rats ${ }^{6}$, many of which have been implicated in neurodegenerative disease, including Parkinson's disease. In the fourth report the Myo5a mutation was associated with marked abnormal hyperphosphorylation of alphasynuclein and tau, and dopamine metabolism alteration, findings also incriminated in the etiopathogenesis of this neuroendocrine/movement disorder in our rat model ${ }^{2}$.

\section{Hypothyroidism neuroendocrine alterations}

The hypothyroid phenotype in our model was believed to be due to an autosomal recessive mutation based on pedigree analysis. ${ }^{5}$. This first report regarding the neuroendocrine disorder was found in a colony of SpragueDawley, which is an outbred white rat. Being a white rat, we were not able to distinguish any abnormality regarding the coat color.

Thyroid hormones (THs) analysis of ataxic and control littermates suggests that the hypothyroid condition in our model was central, most likely "tertiary" (hypothalamic) in origin. This hypothesis was supported by the fact that thyrotropin releasing hormone (TRH) and thyroid stimulating hormone (TSH) were decreased despite the low serum concentration of both $\mathrm{T} 4$ and $\mathrm{T} 3^{5}$.

In our rat model of hypothyroidism there was marked astrogliosis in the CNS, most severe in the cerebellum and hippocampal regions. Astrocytes play an essential role in thyroid hormone metabolism in the brain, being the principal transporter of T4 from the blood and responsible for its conversion to $\mathrm{T} 3$, the biologically active form ${ }^{18,19}$.

Our data along with reports from the literature suggest 
that astrocytosis present in our hypothyroid rats might play a role in observed neuronal alterations. Whether there is a failure of metabolic functions of these proliferating astrocytes due to THs deficiency needs further exploration. The persistence of clusters of neuron-like cells in the adrenal medulla in hypothyroid rats at 25-30 postnatal is interpreted as delayed differentiation.

In summary, in this neonatal inherited tertiary hypothyroidism rat animal model, there were widespread CNS changes with fatal consequences to the host. It appears that we were dealing with a systemic disease cause by a genetic mutation, which affects the homeostatic environment in the body of mutant rats. The discovery of Myo5a mutation, and the neuroendocrine findings previously reported raised some questions regarding their relationship.

a. How does Myo5a mutation relates to endocrine alterations?

Myosin5a is an actin-based motor molecule protein that supports multiple functions within neurons that together encompass a diverse array of fundamental processes that continuously mediate transport of organelles, membranous cargo, secretory vesicles, mRNA, and lipids, emphasizing their roles in neuronal development, axonal transport, dendritic spine structure, and synaptic plasticity ${ }^{1}$.

b. How does this mutation helped us to formulate our hypothesis "delayed neurodevelopment and neurodegeneration associated with Myo5a mutation?

We hypothesize that these phenotypes are due to the absence of Myo5a protein expression in the brain of mutant rats since we do not find any other variants that associate with the mutant phenotype based on WGS data. Our transmission electron microscopy pathology data demonstrated that neuronal degeneration begins as a synaptic event with widespread post-synaptic degeneration and marked accumulation of presynaptic vesicles. The role of actin-based motor Myo5a in co-operation with other molecular motor proteins is to facilitate transynaptic passage for neurotransmitters and grow factors from postsynaptic/dendritic sites into axons/presynaptic sites to exert their normal physiological functions ${ }^{2}$. In the absence of Myo5a in the brain of mutant rats, the synaptic traffic for neurotransmitters and growth factors might be delayed or blocked, triggering the dying-back neuronal degeneration.

\section{Potential role of MicroRNA in the Myo5a rat model of neurodegeneration}

We have shown in our mutant Myo5a rat model data in agreement with the literature on the levels of BDNF ${ }^{21}$ and the effects of miR-132 on Nurr1 expression. We showed that progressive up-regulation of BDNF occurs normally during rat brain development. Interestingly, it is this up-regulation of BDNF during normal development of midbrain dopaminergic neurons that is progressively down-regulated in the mutant rat model. We also observed an increase in expression of micro RNA-132 and a decrease in nuclear receptor related 1 protein (Nurr1). Because BDNF is a direct target of Nurr1, we suggest that mirR132 might regulate Nurr1 levels and thereby influence the development of midbrain dopaminergic neurons ${ }^{6}$. The decline in Nurr1 expression correlates with the loss of tyrosine hydroxylase that we previously reported in this model $^{7}$. In addition, it was reported that decreased Nurr1 protein expression was related with $\alpha$-synuclein aggregation in the substantia nigra $(\mathrm{SN})^{22}$. We did not determine if $\alpha$-synuclein co-localized with this miRNA-132 or other miRNAs that were dysregulated in our Myo5a mutated BD-IV rats ${ }^{6}$. However, these interesting findings raise some questions that need to be addressed to make our findings more relevant for neurodegenerative disorders. As we have shown previously, there are increased levels of $\alpha$-syn/tau-P, which is also observed and implicated to play a pathogenetic role in neurogenerative disorders called synucleino/tauopathies ${ }^{2}$. This raises the question whether there is any link between increases in $\alpha$-syn/tau levels and the expression of miR-132 with the resultant downregulation of BDNF in the mutant rat. Moreover, what causes the up-regulation of miR-132? Is this up-regulation a response of oxidative stress or other stresses induced by $\alpha$-syn/tau accumulation, which progress with age? Is there a time-point correlation with increases in $\alpha$-syn/tau and increases in miR-132 and decreases in Nurr1 and BDNF? So far we have shown a time-point study of BDNF, only. Future study should address whether the highly redox-sensitive dopaminergic neurons and neural stem cells, which appear to respond to the global stress due to accumulation of $\alpha$-syn or other protein aggregates, activate miR-132, thereby shutting down cell cycling Nurr1 and BDNF, which are potent inhibitors of neurotoxic-induced neuronal degeneration to prevent apoptotic cell death during brain development ${ }^{6}$. A recent report suggested that miR-132 negatively regulated the expression of synaptic proteins in the neuronal maturation of embryonic neural stem cells (eNSCs) ${ }^{23}$. Our data strongly suggested that in the absence of Myo5a motor protein neuronal degeneration is triggered by a synaptic disfunction. It seems that Myo5a is necessary for maintaining a functional traffic at the synaptic level during normal CNS development.

Absence of Myo5a protein expression in the brain of affected rats, due to their known or less understood protein/protein interactions, creates a pleiotropic domino-like effect that negatively dysregulates the normal homeostatic environment, resulting in malfunction, and neuronal degeneration. 


\section{References}

1. Hirokawa N. Niwa S. Tanaka Y. Molecular motors in neurons: transport mechanisms and roles in brain function, development, and disease. Neuron. 2010; 68: 610-638.

2. Landrock KK, Sullivan $P$, Martini-Stoica $H$, et al. Pleiotropic neuropathological and biochemical alterations associated with Myo5a mutation in a rat Model. Brain Res. 2018 Jan 15; 1679: 155-170. doi: 10.1016/j.brainres.2017.11.029. Epub 2017 Dec 5.PMID: 29217155.

3. Huang JD1, Brady ST, Richards BW, et al. Direct interaction of microtubule and actin-based transport motors. Nature. 1999; 397: 267-270

4. Bridgman PC. Myosin motor proteins in the cell biology of axons and other neuronal compartments. Results Probl Cell Differ. 2009; 48: 91 105.

5. Stoica G, Lungu G, Xie X, et al. Inherited tertiary hypothyroidism in Sprague-Dawley rats. Brain research. 2007; 1148: 205-216. Doi 10.1016/j.brainres.2007.02.042

6. Lungu G, Stoica G, Ambrus A. MicroRNA profiling and the role of microRNA-132 in neurodegeneration using a rat model. Neuroscience letters. 2013; 553: 153-158. Doi 10.1016/j.neulet.2013.08.001

7. Stoica G, Lungu G, Bjorklund NL, et al. Potential role of alphasynuclein in neurodegeneration: studies in a rat animal model. Journal of neurochemistry. 2012; 122: 812-822. Doi 10.1111/j.14714159.2012.07805.

8. Reissmann M, Ludwig A. Pleiotropic effects of coat colour-associated mutations in humans, mice and other mammals. Seminars in cell \& developmental biology. 2013; 24: 576-586. Doi 10.1016/j. semcdb.2013.03.014

9. Futaki S, Takagishi Y, Hayashi Y, et al. Identification of a novel myosinVa mutation in an ataxic mutant rat, dilute-opisthotonus. Mammalian genome : official journal of the International Mammalian Genome Society. $2000 ; 11:$ 649-655.

10. Griscelli C, Prunieras M. Pigment dilution and immunodeficiency: a new syndrome. International journal of dermatology. 1978; 17: 788-791.

11. Pastural E, Barrat FJ, Dufourcq-Lagelouse R, et al. Griscelli disease maps to chromosome $15 \mathrm{q} 21$ and is associated with mutations in the myosin-Va gene. Nature genetics. 1997; 16: 289-292. Doi 10.1038/ ng0797-289
12. Menasche G, Pastural E, Feldmann J, et al. Mutations in RAB27A cause Griscelli syndrome associated with haemophagocytic syndrome. Nature genetics. 2000; 25: 173-176. Doi 10.1038/76024

13. Brooks SA, Gabreski N, Miller D, et al. Whole-genome SNP association in the horse: identification of a deletion in myosin Va responsible for Lavender Foal Syndrome. PLoS genetics. 2010; 6: e1000909. Doi 10.1371/journal.pgen.1000909

14. Mercer JA, Seperack PK, Strobel MC, et al. Novel myosin heavy chain encoded by murine dilute coat colour locus. Nature. 1991; 349: 709713. Doi $10.1038 / 349709 \mathrm{a} 0$

15. Takagishi Y, Oda S, Hayasaka S, et al. The dilute-lethal (dl) gene attacks a Ca2+ store in the dendritic spine of Purkinje cells in mice. Neuroscience letters. 1996; 215: 169-172.

16. Dekker-Ohno K, Hayasaka S, Takagishi Y, et al. Endoplasmic reticulum is missing in dendritic spines of Purkinje cells of the ataxic mutant rat. Brain research. 1996; 714: 226-230.

17. Takagishi Y, Murata Y. Myosin Va mutation in rats is an animal model for the human hereditary neurological disease, Griscelli syndrome type 1. Annals of the New York Academy of Sciences. 2006; 1086: 6680. Doi 10.1196/annals.1377.006

18. Ohno K, Kanou Y, Oda S, et al. Mapping of the dilute-opisthotonus (dop) gene on chromosome 8 of the rat. Experimental animals / Japanese Association for Laboratory Animal Science. 1996; 45: 71-75.

19. Trentin AG. Thyroid hormone and astrocytes morphology J.Endocrinol. 2006 May; 189(2): 189-97. Review.

20. Clos J, Lagrand J. Effects of thyroid deficiency on different cell populations of cerebellum in young rats. Brain Research. 1973; 63: 450-455.

21. Scalzo P, Kümmer A, Bretas TL, et al. Serum levels of brain-derived neurotrophic factor correlate with motor impairment in Parkinson's disease. J Neurol. 2010 Apr; 257(4): 540-5. doi: 10.1007/s00415009-5357-2. Epub 2009 Oct 22.

22. Gao FB. Posttranscriptional control of neuronal development by miRNA networks, Trends in Neurosciences. 2008; 31: 20-26.

23. Aya Yoshimura, Tadahiro Numakawa, Haruki Odaka, et al. Negative regulation of microRNA-132 in expression of synaptic proteins in neuronal differentiation of embryonic neural stem cells. Neurochemistry International. 2016; 97: 26e33. 